\title{
Spatial frequency and attention: Effects of level-, target-, and location-repetition on the processing of global and local forms
}

\author{
MARVIN R. LAMB \\ California State University, Hayward, Califormia \\ and Veterans Administration Medical Center, Martinez, California \\ and \\ E. WILLIAM YUND \\ Veterans Administration Medical Center, Martinez, California
}

\begin{abstract}
Is attentional selection between local and global forms based on spatial frequency? This question was examined by having subjects identify local or global forms of stimuli that had been "contrast balanced," a technique that eliminates low spatial frequencies. Response times (RTs) to global (but not local) forms were slowed for contrast-balanced stimuli, suggesting that low spatial frequencies mediate the global RT advantage typically reported. In contrast, the beneficial effect of having targets appear at the same, as opposed to a different, level as that on the immediately preceding trial was unaffected by contrast balancing. This suggests that attentional selection between different levels of structure is not based on spatial frequency. The data favor an explanation in terms of "priming," rather than in terms of adjustments in the diameter of an attentional "spotlight."
\end{abstract}

Numerous investigators have suggested that the analysis of hierarchically organized stimuli might depend on the differences in spatial frequency between local and global forms (Badcock, Whitworth, Badcock, \& Lovegrove, 1990; Hughes, Fendrich, \& Reuter-Lorenz, 1990; LaGasse, 1993; Lamb \& Robertson, 1990; Lovegrove \& Pepper, 1994; Navon, 1991; Sergent, 1982, 1987; Shulman, Sullivan, Gish, \& Sakoda, 1986). Local information (e.g., the $\mathrm{Ss}$ in Figure la) is carried in relatively high spatial frequencies, whereas global information (e.g., the A in Figure 1a) is carried in relatively low spatial frequencies. Several studies have provided evidence that the speed or efficiency with which local and global levels of structure are analyzed depends on these differences in spatial frequency (LaGasse, 1993; Lamb \& Yund, 1993, in press; Badcock et al., 1990; Shulman et al., 1986). For example, Shulman et al. adapted subjects to sinusoidal gratings of different spatial frequencies and then had them identify local or global targets. The adapting frequency that most affected performance on the global task

This research was supported by the Medical Research Council of the Department of Veterans Affairs and by National Institute of Neurological Disorders and Stroke Grant NS27902 to M.R.L. We would like to thank Russell L. De Valois for allowing us to use his equipment to perform the Fourier transforms of the stimuli. We would also like to thank Lester Krueger, Stephen Christman, and an anonymous reviewer for their helpful suggestions. Finally, we would like to thank Betsy London and Kelly Jorgensen for their help with collecting data. Correspondence concerning this article should be addressed to M. R. Lamb, Department of Psychology, California State University, Hayward, CA 94542 (e-maii.mlamb@csuhayward.edu) was lower than the adapting frequency that most affected performance on the local task.

The role that spatial frequency might play in the analysis of hierarchical structure is complicated by recent neuropsychological evidence suggesting that several interacting but separate mechanisms are involved in the analysis of hierarchically organized stimuli (Lamb, Robertson, \& Knight, 1989, 1990; Robertson \& Lamb, 1991; Robertson, Lamb, \& Knight, 1988). These studies have demonstrated lesion-site-specific selective impairment of (1) the speed or efficiency with which targets at different levels of structure are identified (referred to as reaction time, or RT, advantage), (2) the degree to which distractors at one level affect responding to targets at another (referred to as interference), and (3) changes in RTs occurring in response to changing task demands (referred to as attention). Similar dissociations have been demonstrated in neurologically intact subjects as well (LaGasse, 1993; Lamb \& Robertson, 1989; Lamb \& Yund, 1993; Lovegrove \& Pepper, 1994; Navon \& Norman, 1983; Paquet, 1992). To the extent that several different mechanisms are involved in the analysis of hierarchically organized stimuli, it becomes necessary to determine the role that spatial frequency plays for each mechanism, because it is quite possible that spatial frequency is important to the functioning of some mechanisms but not others. Indeed, a recent study by Lamb and Yund (1993) provided evidence that spatial frequency information was important for RT advantage but not for interference or attention.

Our main purpose in the present experiment was to explore further the possible role that spatial frequency 


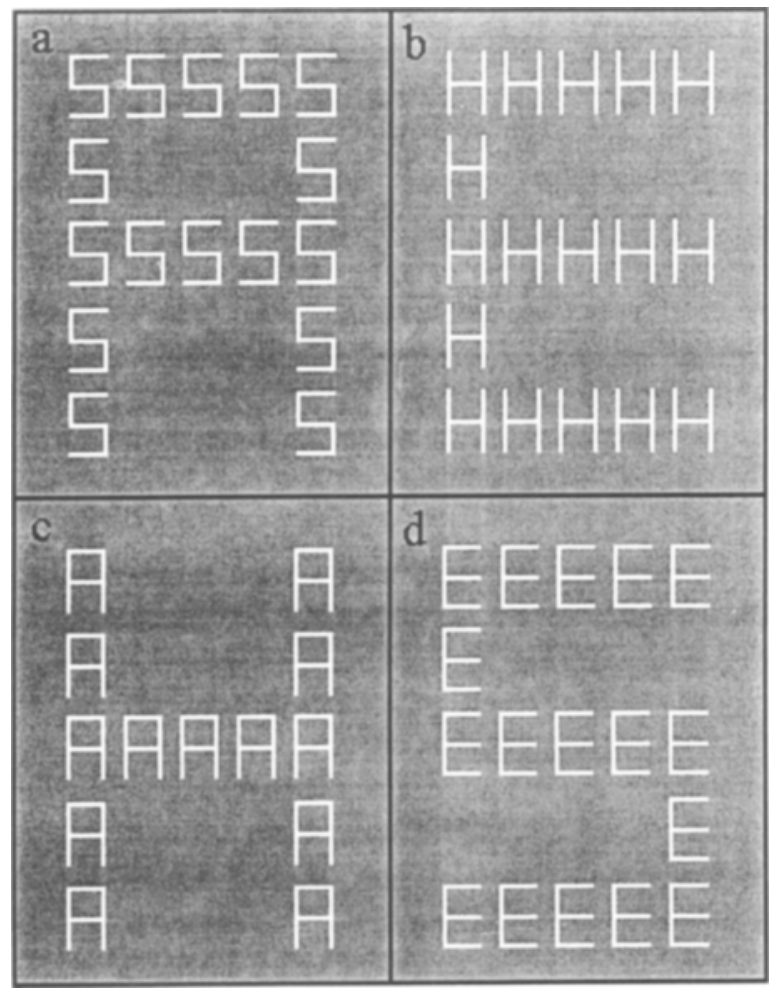

Figure 1. Drawings of four (of the eight) hierarchical letter patterns used in the present experiments. Shown are a global "A" composed of local " $S$ "s (a), a global " $E$ " composed of local " $H$ "s (b), a global " $H$ " composed of local " $A$ "s (c), and a global "S" composed of local "E"'s (d).

might play in the ability to shift attention between local and global levels of structure. Several investigators have provided evidence that the analysis of different levels of structure is subject to attentional control (Kinchila, SolisMacias, \& Hoffman, 1983; Lamb \& Robertson, 1988; Lamb \& Yund, 1993; Miller, 1981; Pomerantz, 1983; Robertson, Egly, Lamb, \& Kerth, 1993; Ward, 1982). Furthermore, Shulman and Wilson (1987) have provided evidence that such attentional control might be based on selection between, or differential weighting of, high versus low spatial frequency channels. They induced subjects to attend selectively to different levels of structure by having them identify local or global forms in separate blocks of trials. On a small number of probe trials, subjects were also asked to detect sine wave gratings of different spatial frequencies. Low-frequency gratings were more easily detected than high-frequency gratings in the context of the global task, while the reverse was true in the context of the local task. This is consistent with the notion that attention to level is based on spatial frequency.

More recently, however, Lamb and Yund (1993) showed that attentional selection between different levels of structure can occur in the absence of a difference in spatial frequency between local and global forms. In their experiments, hierarchically organized stimuli contained one target letter and one distractor letter. In one block of trials (local bias), targets were more likely to ap- pear at the local level, and in another block (global bias), targets were more likely to appear at the global level. Subjects identified which target letter was present in the stimulus, regardless of the level at which it appeared. In this "biasing" procedure, a global RT advantage is found in the global bias condition, whereas a local RT advantage is found in the local bias condition. It has been argued that this performance tradeoff reflects shifts of attention that occur in response to the changing task demands. Lamb and Yund compared performance with typical broadband hierarchically organized stimuli in which information about different levels of structure is carried by different spatial frequencies to performance with stimuli that had been "contrast balanced." Contrast balancing selectively eliminates low spatial frequencies so that both local and global forms must be identified on the basis of high spatial frequencies. They found that although contrast-balancing increased RTs to global targets overall, it had no effect on the performance tradeoff indicative of attentional control. These data led the authors to suggest that spatial frequency is not an important factor in attentional selection between hierarchical levels of structure.

In the present experiment, we explored the possibility that the failure of Lamb and Yund (1993) to find an effect of spatial frequency on attentional selection might have been due to the measure that they used. This "biasing" procedure produces a robust performance tradeoff, which has been taken as a measure of subjects' ability to shift attention between levels of structure. However, recent evidence suggests that two separate attentional mechanisms can modulate the analysis of global and local level information (Lamb \& Robertson, 1988; Robertson et al., 1993). First, changes in the diameter and/or location of a spatial attentional "spotlight" may differentially affect the analysis of local and global forms. This has been referred to as "regional" attention. Second, some attribute that distinguishes local from global forms might serve as a basis for selecting information at a given level of structure for preferential analysis. This has been referred to as "categorical" attention. Robertson et al. (1993) suggested that spatial frequency was the attribute most likely to be the basis for "categorical" selection. Perhaps Lamb and Yund failed to find an effect of spatial frequency because their "biasing" procedure tapped the regional rather than the "categorical" mechanism.

A different measure of attention, which depends on the sequence of local and global level targets within a block of trials, has been used by several investigators (Filoteo et al., 1994; Filoteo, Delis, Massman, Demadura, \& Butters, 1992; Ward, 1982). Here, a comparison is made between performance on trials in which the target is at the same level as on the immediately preceding trial (repeated-level trials) and performance on trials in which the target level has changed from the previous trial (changed-level trials). With this procedure, RTs have been found to be faster on repeated-level than on changed-level trials, presumably reflecting the benefit of 
attention being directed to the relevant level on trial $n$ as the result of just having analyzed a target at that same level on trial $n-1$. While Ward (1982) originally interpreted such repetition effects in terms that would be consistent with "regional" attention, Robertson et al. (1993) argued from their data that such effects were the result of "categorical" selection and were likely to be based on spatial frequency.

In the present experiment, we examined how performance on repeated-level and changed-level trials is affected when low spatial frequencies are removed from the stimulus. If level-repetition effects are based on spatial frequency, this should reduce or eliminate the difference between repeated-level and changed-level trials. Subjects received two different types of stimuli. The first type consisted of typical broadband stimuli, with high spatial frequencies carrying information about both local and global forms, as well as low spatial frequencies carrying information about global but not local forms. These stimuli appeared simply as white letters on a gray background (see Figure 2a) and will be referred to here as bright stimuli (after Hughes et al., 1990). The second type was identical to the first, except that each local letter was surrounded by a dark outline (see Figure $2 b$ ). These will be referred to as contrast-balanced stimuli. Contrast balancing eliminates low spatial frequencies that specify global letters. Thus, for contrast-balanced stimuli, both local and global letters must be identified on the basis of high spatial frequencies. If level-repetition effects are based on subjects categoriz-ing local and global forms as high versus low spatial frequency, respectively, the difference between repeated-level and changed-level trials should be reduced or eliminated for contrast-balanced stimuli.

It is possible, however, that a level-repetition effect might reflect the operation of a "regional" rather than a "categorical" selection mechanism. For example, it has been suggested (Lamb \& Robertson, 1988, 1990; Ward, 1982) that efficient analysis of local and global targets occurs when the size of the attended area conforms to the size of the target form (i.e., is relatively large for global and small for local targets). If so, the benefit of level rep-

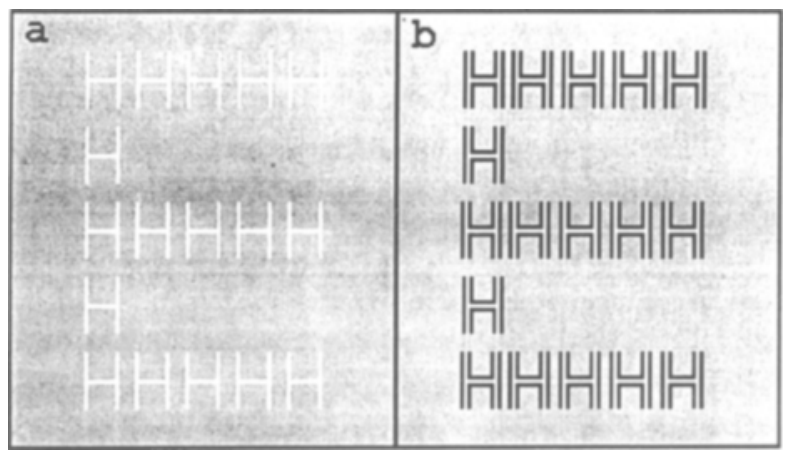

Figure 2. Drawings of a bright stimulus (a) and a contrastbalanced stimulus (b). The precise spatial frequency content of the stimuli depends critically on luminance levels, which are not accurately reproduced in this figure. See text for details. etition might derive from adjustments in the diameter of the attended area being carried over from trial $n-1$ to trial $n$. However, it would seem that this benefit should occur only (or at least much more so) when the stimulus also occurs at the same location on trials $n$ and $n-1$. This is because having the size of the spotlight adjusted appropriately would seem to be of value only if the spotlight is directed at the correct location as well. In the present experiment, the level of the target and the location (or visual field) of the target were varied independently. If level repetition facilitates performance because adjustments in the size of the attended area carry over from one trial to the next, one would expect to observe the level-repetition effect only on trials on which visual field repetition also occurred.

Another possibility is that level repetition might produce faster RTs for reasons having more to do with mechanisms of response selection than with how hierarchical stimuli are processed. Several investigators have provided evidence that subjects have a tendency to "bypass" normal processing of the stimulus and to select their response on the basis of whether or not the stimulus matches or mismatches the one presented on the previous trial. Specifically, subjects are biased to make the same response as that given on the previous trial if the stimulus is repeated, but to change their response if some attribute of the stimulus changes from that seen on the previous trial, even if that attribute is irrelevant to the task (Bertelson, 1965; Fletcher \& Rabbitt, 1978; Krueger \& Shapiro, 1981; Terry, Valdes, \& Neill, 1994).

This "bypass rule" predicts that level repetition will have opposite effects, depending on whether or not the same response is required on trials $n-1$ and $n$. When the same response is required on trials $n-1$ and $n$, RTs should be relatively fast when the target level is also repeated, because of the tendency to (correctly) repeat the response when the stimulus is repeated. Conversely, RTs should be relatively slow when the target level changes from that on the previous trial, because the change in the stimulus will engender a tendency to (incorrectly) change responses even though the same response is called for. This pattern should be reversed when different responses are required on trials $n-1$ and $n$. In this case, level repetition should actually harm performance because the tendency will be to (now incorrectly) repeat the same response when the level is repeated but to (now correctly) change the response when the level changes.

Another purpose of the present experiment was to examine the effects of spatial frequency on interference between local and global level information. In his seminal work, Navon (1977) found that global distractors interfered with (i.e., slowed) responses to local targets but that local distractors had no effect on responses to global targets. This asymmetric global interference was taken as evidence that global analysis precedes local analysis. Numerous subsequent studies have replicated Navon's findings, although interference is not always asymmetric. Recently, a number of investigators have proposed that such interference effects reflect the effects of inhibitory 
interactions among high versus low spatial frequency channels (Hughes, 1986; Hughes et al., 1990; Hughes, Layton, Baird, \& Lester, 1984; Kitterle, Christman, \& Conesa, 1993). However, Lamb and Yund (1993, in press) have found that interference is unaffected by contrast balancing. The fact that interference occurred even when both local and global information were carried in high spatial frequencies is not consistent with the hypothesis that interference between information at different levels of structure results from inhibitory interactions between high and low spatial frequency channels. The present experiment provided an opportunity to replicate this finding.

Each stimulus pattern in the present experiment (see Figure 1) contained one target letter (" $\mathrm{H}$ " or "S") and one distractor letter ("A" or "E"). Several studies have shown that under certain conditions, RTs to " $\mathrm{H}$ " targets are faster when the distractor is an " $A$ " than when it is an "E," and RTs to " $S$ " targets are faster when the distractor is an " $E$ " than when it is an " $A$ " (Lamb \& Robertson, 1989; Lamb et al., 1990; Lamb \& Yund, 1993). When this target letter $\times$ distractor letter interaction is present, it shows that distractors at one level of structure are affecting responding to targets at the other, and this is the measure of between-level interference used in the present study. If this interference is produced by inhibitory interactions between high and low spatial frequency channels, the target letter $\times$ distractor letter interaction should be reduced or eliminated for contrast-balanced stimuli.

The present experiment also provided an opportunity to examine a hypothesis offered by Previc (1990) that the upper visual field is specialized for local analysis, whereas the lower visual field is specialized for global analysis. Consistent with this hypothesis, Christman (1993) found an interaction between visual field of stimulus presentation (upper vs. lower) and target level (local vs. global) in the direction predicted by Previc. However, as Christman pointed out, the visual field advantage in these experiments was not always significant in absolute terms (e.g., the global RT advantage for stimuli presented in the lower visual field was significant in only one of his two experiments). He suggested that this might reflect (1) insufficient power due to a relatively small number of trials per condition and/or (2) the fact that stimulus location was more uncertain than in typical laterality experiments (i.e., stimuli were presented randomly in four locations rather than two). The present experiment provided an opportunity to examine whether differences in performance between the upper and lower visual fields are found when these conditions are not present.

\section{METHOD}

\section{Subjects}

The 19 female and 13 male subjects ranged in age from 18 to 32 years $(M=21.0, S D=3.69)$. They received course credit at $\mathrm{Cal}$ ifornia State University, Hayward for their participation and gave informed consent. All were right-handed and reported having normal or corrected-to-normal visual acuity.

\section{Apparatus}

The stimuli were generated on an NEC 3FGe color monitor controlled by an 80486-based microcomputer (IBM compatible) and a VGA graphics card. Stimulus timing (onset, termination, and duration) was tied to the vertical synchronization pulse (refresh rate, approximately $60 \mathrm{~Hz}$ ). All other events (responses, intertrial interval, or ITI, etc.) were timed with the 8253 chip set to a 1 -msec time base. The status of the response keys (two push-button microswitches of a Gravis game controller) was monitored via the game port.

\section{Stimuli}

Global letters were constructed from local letters in a $5 \times 5$ matrix. Global letters were $3.2^{\circ}$ high and $2.0^{\circ}$ wide. Local letters were $.44^{\circ}$ high and $.27^{\circ}$ wide. The letters " $\mathrm{H}$ " and " $\mathrm{S}$ " served as targets, and the letters " $A$ " and "E" served as distractors. Each stimulus pattern contained one target and one distractor letter, resulting in eight stimuli (four of which are shown in Figure 1).

All stimuli were presented on a gray $\left(46 \mathrm{~cd} / \mathrm{m}^{2}\right)$ background. Bright stimuli (see Figure 2a) were composed of lines that were brighter $\left(66.5 \mathrm{~cd} / \mathrm{m}^{2}\right)$ than the background. The contrast-balanced stimuli (see Figure 2b) were identical to the bright stimuli, except that the bright lines composing each local letter were surrounded by lines that were darker $\left(36.1 \mathrm{~cd} / \mathrm{m}^{2}\right)$ than the background. The luminance levels of the bright and dark lines were chosen so that the space-averaged luminance of the contrast-balanced stimuli would equal that of the background. Thus, the change in luminance from background was approximately twice as great for bright lines as for dark lines, because dark lines occupied twice as much area as did bright ones (see Figure $2 \mathrm{~b}$ ). All lines (both bright and dark) were approximately $.009^{\circ}$ thick.

A two-dimensional Fourier transformation of representative bright and contrast-balanced stimuli was performed (see Figure 3). Figure $3 \mathrm{a}$ shows the transform for the bright stimulus shown in Figure 2a. As can be seen, bright stimuli contained a broad spectrum of spatial frequencies, with power concentrated near horizontal and vertical orientations. Figure $3 b$ shows the transform for the contrast-balanced stimulus shown in Figure $2 \mathrm{~b}$. This figure shows that the contrast-balanced stimuli had much less power at low spatial frequencies than did the bright stimuli. More specifically, the contrast-balanced stimuli had virtually no power at spatial frequencies below 3 cycles/deg (i.e., the area within the inner circle in Figure 3 ) and very little power even out as far as $6 \mathrm{cy-}$ cles/deg (i.e., the area between the two circles in Figure 3). Figure 3 represents a very conservative estimate of the effect of contrast balancing. All spatial frequencies for a given stimulus with contrast above .0006 are plotted. Since threshold for all spatial frequencies is above .001 at these luminance levels (see DeValois \& DeValois, 1990), all above-threshold spatial frequencies contained in the stimulus are plotted in Figure 3, and in fact some of the plotted points are well below threshold. The effect of contrast balancing illustrated in Figure 3 is representative of the effect of contrast balancing for all the stimuli used in this experiment.

A small $\left(.26^{\circ}\right)$ black $\left(16 \mathrm{~cd} / \mathrm{m}^{2}\right)$ square presented in the center of the screen served as the fixation point. All luminance measurements were taken with a Minolta CS-100 Chroma Meter. The experiment was conducted with ordinary overhead room lighting.

\section{Procedure}

The distance between the subject and the monitor screen was fixed at $65 \mathrm{~cm}$ by the use of a chinrest head restraint. Each trial began with a $500-\mathrm{msec}$ tone, followed immediately by a $500-\mathrm{msec}$ presentation of the central fixation point. Subjects were instructed to look directly at the fixation point and not to move their eyes. The fixation point was followed immediately by a $100-\mathrm{msec}$ presentation of one of the stimulus patterns, which appeared randomly, but equally often, above or below the fixation point $\left(1.2^{\circ}\right.$ from fixation to the nearest edge of the stimulus pattern). There was a $1-\mathrm{sec}$ ITI. 


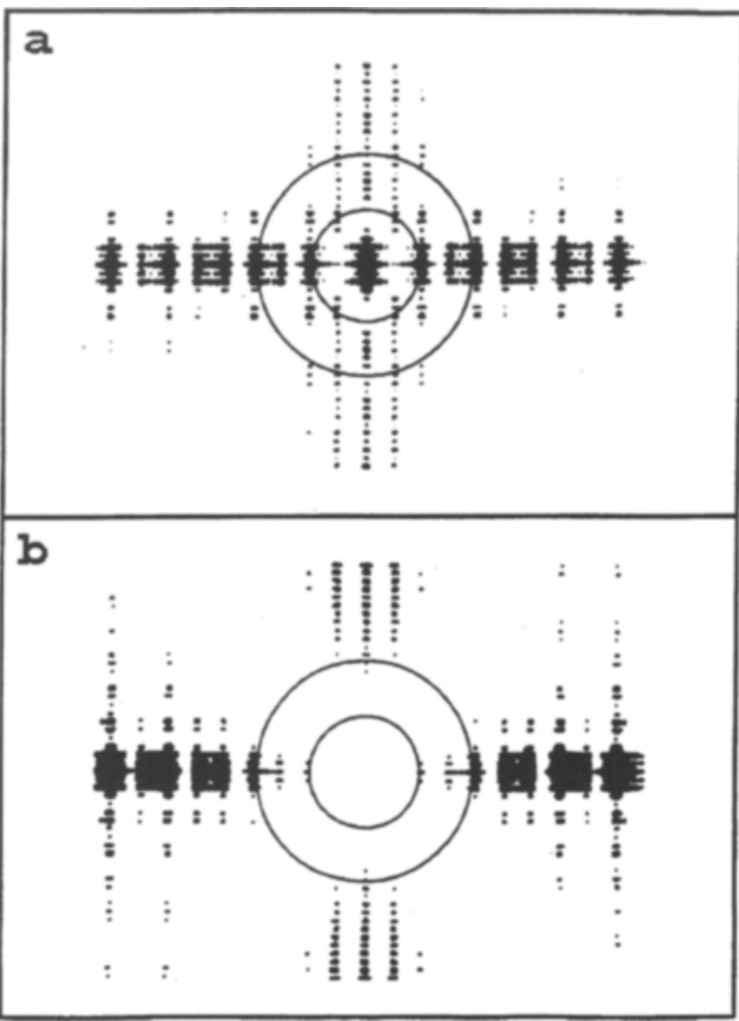

Figure 3. Two-dimensional Fourier transformations of the stimuli shown in Figure 2. Figure 3a shows the transform for the bright stimulus shown in Figure 2a. Figure 3b shows the transform for the contrast-balanced stimulus shown in Figure $2 b$. The transforms are plotted in polar coordinates, with spatial frequency on the radial dimension and orientation on the angular dimension. Spatial frequency increases linearly from zero at the center. The radii of the inner and outer circles are at 3 and 6 cycles/ deg, respectively. The horizontal meridian of the polar coordinates indicates horizontal spatial frequency components, and the vertical meridian indicates vertical spatial frequency components. The size of each plotted point indicates the power of a given spatial frequency at a given orientation. When stimuli contain substantial power at many similar spatial frequencies and orientations, plotted points overlap and produce larger filled areas.

Subjects used their dominant hand to indicate which of the target letters ("H" or "S") was present on each trial. The subjects, who were right-handed, pressed the " $\mathrm{H}$ " key with their index finger and the "S" key with their middle finger. Subjects were instructed to respond as quickly as possible while minimizing errors.

Each subject received eight blocks of 131 trials with a short rest break between each block. The first 3 trials of each block were considered warm-up trials and were not included in the analysis. Each block contained all bright or all contrast-balanced stimuli. Subjects alternated between bright and contrast-balanced stimuli every two blocks. Half of the subjects began with bright stimuli and the other half with a contrast-balanced stimuli. Target level (local and global), target letter (" $\mathrm{H}$ " and "S"), distractor letter ("A" and " $E$ "), and stimulus location (above and below fixation) were completely counterbalanced within each block. The stimuli were presented randomly, except that the target could not appear at the same level, or be the same letter, or appear in the same location, on more than four consecutive trials. Four different randomizations were generated. Each subject received Randomization ! for Blocks
1 and 3, Randomization 2 for Blocks 2 and 4, Randomization 3 for Blocks 5 and 7, and Randomization 4 for Blocks 6 and 8 . This resulted in subjects' receiving the same randomizations for both bright and contrast-balanced stimuli. Subjects also received two 16-trial practice blocks, one with bright stimuli and one with contrast-balanced stimuli, before data collection began.

Repeated-level and changed-level trials were determined by examining the sequence of trials that a given randomization had produced. Repeated-level trials were defined as any trial $(n)$ in which the preceding trial $(n-1)$ had contained a target at the same level. Changed-level trials were defined as any trial in which targets occurred at different levels on trials $n$ and $n-1$. Repeated-target trials were defined as any trial in which the same target letter occurred on trials $n$ and $n-1$. Changed-target trials were defined as any trial in which different target letters occurred on trials $n$ and $n-1$. Repeated-field trials were defined as any trial in which the target occurred in the same location on trials $n$ and $n-1$. Changedfield trials were defined as any trial in which the target occurred in different locations on trials $n$ and $n-1$.

\section{RESULTS}

The data (both RTs and errors) were subjected to an analysis of variance (ANOVA) with visual field (upper and lower), frequency content (bright or contrast balanced), target level (local and global), target letter $(\mathrm{H}$ and $S$ ), and distractor letter ( $A$ and $E$ ) as repeated measures factors. Median RTs (correct trials only) were calculated for each cell in the design for each subject, and the RT data reported are means of those medians. Mean error rates and RTs for each cell in the design are given in Table 1.

\section{Errors}

The overall error rate was low $(M=5.7 \%)$. There was a main effect of frequency content $[F(1,31)=78.64, p<$ $.001]$, reflecting the fact that fewer errors were made when the target was bright than when it was contrast balanced. There was a main effect of target $[F(1,31)=$ $26.64, p<.001$ ], reflecting the fact that fewer errors were made when the target was an " $H$ " than when it was an "S." There was also a frequency content $\times$ target level $\times$ target letter interaction $[F(1,31)=9.40, p<.01\}$. This was because the advantage for " $\mathrm{H}$ " targets was greater for local than for global targets when the stimulus was bright, but greater for global than for local targets when the stimulus was contrast balanced. The frequency content $X$ visual field $\times$ target letter interaction was significant $[F(1,31)=9.28, p<.01]$. This was because the advantage for " $\mathrm{H}$ " targets was greater for stimuli in the upper visual field than in the lower when the stimulus was bright, but greater for stimuli in the lower visual field than in the upper when the stimulus was contrast balanced. There was a main effect of distractor letter $[F(1,31)=14.02, p<.001]$, reflecting the fact that fewer errors were made when the distractor was an "E" than when it was an "A." The target letter $\times$ distractor letter interaction was also significant $[F(1,31)=36.54, p<$ $.001]$, reflecting the fact that fewer errors occurred when the distractor was an "E" than when it was an " $A$ " if the target was an "S," but fewer errors occurred when the 
Table 1

Mean Response Time (in Milliseconds) and Percentage Errors for Each Target and Distractor Letter as a Function of Stimulus Type and Visual Field

\begin{tabular}{|c|c|c|c|c|c|c|c|c|c|}
\hline \multirow[b]{3}{*}{ Target } & \multirow[b]{3}{*}{ Distractor } & \multicolumn{4}{|c|}{ Bright } & \multicolumn{4}{|c|}{ Contrast Balanced } \\
\hline & & \multicolumn{2}{|c|}{ Upper Field } & \multicolumn{2}{|c|}{ Lower Field } & \multicolumn{2}{|c|}{ Upper Field } & \multicolumn{2}{|c|}{ Lower Field } \\
\hline & & RT & Errors & RT & Errors & RT & Errors & RT & Errors \\
\hline \multirow[t]{2}{*}{ Global H } & A & 584 & 4.1 & 555 & 2.7 & 612 & 4.7 & 573 & 6.2 \\
\hline & $\mathbf{E}$ & 583 & 3.9 & 546 & 2.3 & 643 & 6.3 & 594 & 4.6 \\
\hline \multirow[t]{2}{*}{ Global S } & A & 605 & 7.4 & 638 & 7.3 & 671 & 12.0 & 673 & 10.1 \\
\hline & $E$ & 576 & 3.0 & 552 & 3.4 & 646 & 6.7 & 628 & 6.6 \\
\hline \multirow[t]{2}{*}{ Local H } & A & 532 & 3.6 & 515 & 2.2 & 544 & 4.6 & 535 & 5.9 \\
\hline & $\mathrm{E}$ & 555 & 4.2 & 568 & 4.4 & 575 & 6.9 & 574 & 9.9 \\
\hline \multirow[t]{2}{*}{ Local S } & A & 607 & 6.7 & 637 & 10.0 & 633 & 11.6 & 627 & 7.5 \\
\hline & $\mathrm{E}$ & 584 & 5.0 & 599 & 6.3 & 575 & 6.3 & 602 & 5.1 \\
\hline
\end{tabular}

distractor was an "A" than when it was an " $E$ " if the target was an " $H$." The frequency content $X$ target letter $X$ distractor letter interaction was also significant $[F(1,31)=14.97, p<.001]$, reflecting the fact that the target letter $\times$ distractor letter interaction was greater for contrast-balanced than for bright stimuli.

\section{Reaction Time}

There was a significant main effect of target level $[F(1,31)=5.28, p<.05]$, reflecting the fact that RTs were faster for local than for global targets. There was also a main effect of frequency content $[F(1,31)=$ $14.48, p<.001]$, reflecting the fact that RTs were faster for bright than for contrast-balanced stimuli. However, there was also a significant frequency content $\times$ target level interaction $[F(1,31)=77.10, p<.001]$, reflecting the fact that contrast balancing slowed responses to global $[F(1,31)=40.03, p<.001]$, but not to local $[F(1,31)=1.08]$, targets (see Figure 4 ).

There was also evidence of interference between forms at different levels. There was a significant target letter $\times$ distractor letter interaction $[F(1,31)=44.74$, $p<.001]$, reflecting the fact that RTs were faster when the distractor was an " $A$ " than when it was an " $E$ " if the target was an "H," but faster when the distractor was an " $E$ " than when it was an " $A$ " if the target was an " $S$ " (see Figure 5). The target level $\times$ target letter $\times$ distractor letter interaction was nonsignificant $(F<1)$; thus there

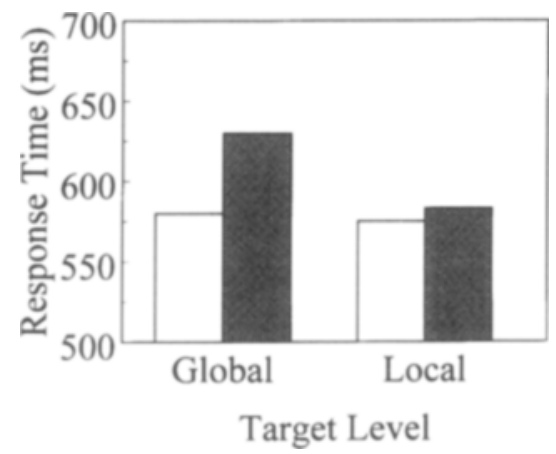

Figure 4. Mean response time (in milliseconds) to global and local targets for bright (open bars) and contrast-balanced (filled bars) stimuli. was no indication that interference was asymmetric. There was also no indication that contrast balancing reduced interference. The frequency content $\times$ target letter $\times$ distractor letter interaction was nonsignificant $(F<1)$, and the target letter $\times$ distractor letter interaction was significant for contrast-balanced stimuli $[F(1,31)=61.54, p<.001]$ as well as for bright ones $[F(1,31)=22.47, p<.001]$.

Responses were faster in the lower visual field than in the upper visual field for global stimuli $[F(1,11)=$ $11.66, p<.01$ ], but not for local stimuli $(F<1)$ (see Figure 6). This interaction did not quite obtain statistical reliability $[F(1,31)=3.48, p<.07]$, but the means are in the same direction as that found by Christman (1993) and predicted by Previc (1990). In addition, several factors interacted with visual field. There was a significant frequency content $\times$ visual field interaction $[F(1,31)=$ $6.68, p<.05]$, reflecting the fact that contrast balancing had a somewhat more deleterious effect for stimuli presented above fixation. There was also a visual field $\times$ target letter $\times$ distractor letter interaction $[F(1,31)=4.55$, $p<.05$ ], reflecting the fact that interference was somewhat less for stimuli presented in the upper visual field than for those presented in the lower visual field (see Figure 5). Finally, the visual field $\times$ frequency content $\times$ target letter $\times$ distractor letter interaction was significant $[F(1,31)=11.24, p<.01]$, reflecting the fact that contrast balancing had little or no effect on interference for stimuli in the lower visual field, but slightly increased interference in the upper visual field $[F(1,31)=$ $6.68, p<.05$ ] (see Figure 5).

\section{EFFECTS OF REPETITION}

Since trial sequence was randomly determined, each of the 32 different trial types (i.e., two frequency contents, two visual fields, two levels, two targets, and two distractors) was not necessarily equally represented for each repetition condition (i.e., repeated or changed level, repeated or changed target, and repeated or changed field) in the 1,024 data trials that each subject received. Thus, in order to accurately evaluate the effects of repetition, the first instance (correct trials only for RT data) of each of the 256 possible trial types (all the factors 


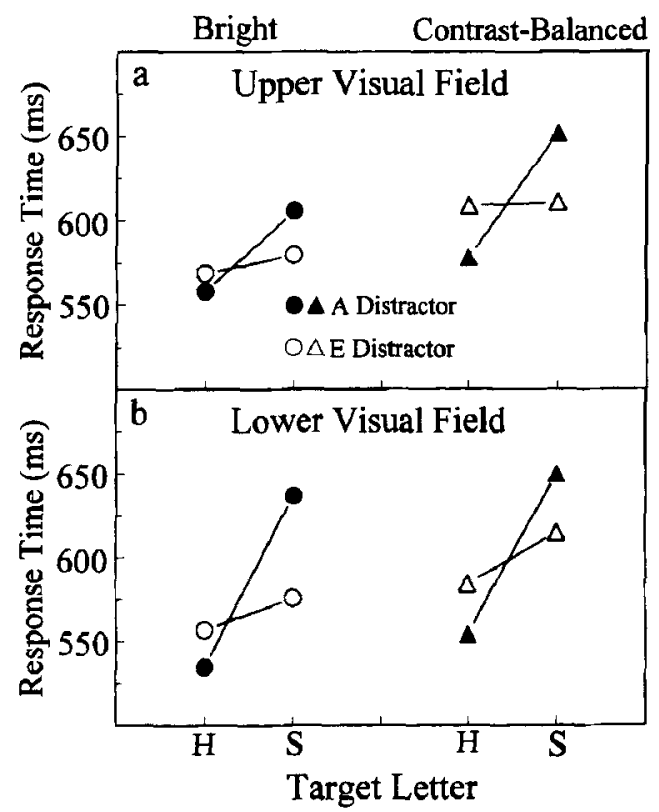

Figure 5. Mean response time (in milliseconds) to " $H$ " and " $S$ " targets when the distractor was an " $A$ " (closed symbols) or an "E" (open symbols). Data are presented separately for bright stimuli (circles) and contrast-balanced stimuli (triangles) and for stimuli presented in the upper visual field (a) and lower visual field (b).

listed above plus the three repetition factors) was selected for each subject for the analysis of repetition effects. This ensured that each trial type was equally represented for each repetition condition. These data (both RT and errors) were subjected to an ANOVA with frequency content (bright and contrast balanced), target level (global and local), level repetition (repeated level and changed level), target repetition (repeated target and changed target), and field repetition (repeated field and changed field) as repeated measures factors. The means for each cell in these analyses are presented in Table 2.

\section{Errors}

The overall error rate was low $(M=5.9 \%)$. There was a main effect of frequency content $[F(1,31)=85.37, p<$ $.001]$, reflecting the fact that fewer errors were made when the target was bright than when it was contrast balanced. There was a main effect of target level $[F(1,31)=$ $7.48, p<.01]$, reflecting the fact that fewer errors were made when the target was global than when it was local. There was a main effect of level repetition $[F(1,31)=$ $15.38, p<.001$ ], reflecting the fact that fewer errors were made on repeated-level than on changed-level trials. There was a main effect of target repetition $[F(1,31)=$ $19.42, p<.001]$, reflecting the fact that fewer errors were made on changed-target than on repeated-target trials. The level repetition $\times$ target repetition interaction approached significance $[F(1,31)=3.26, p<.08]$; the level-repetition effect tended to be larger when the target also repeated than when it did not. The target level $X$ level repetition $\times$ target repetition interaction was significant $[F(1,31)=6.25, p<.05]$. This interaction reflects the fact that for global targets, the size of the levelrepetition effect tended to be larger when the target letter also repeated than when it did not, while the reverse was true for local targets. The main effect of field repetition was significant $[F(1,31)=4.7, p<.05]$. Performance was better on repeated-field than on changed-field trials. Finally, there was a significant field repetition $\times$ target repetition interaction $[F(1,31)=27.49, p<.001]$. This was because there were fewer errors on repeated-target than on changed-target trials when the visual field also repeated, but more errors on repeated-target than on changed-target trials when the visual field changed.

\section{Reaction Time}

There was a main effect of level repetition $[F(1,31)=$ $35.20, p<.001]$, reflecting the fact that RTs were faster on repeated-level than on changed-level trials (see Figure $7 \mathrm{a}$ ). There was no indication that the level-repetition effect differed depending on whether repetition occurred at the local or at the global level. Repeated-level trials were $31 \mathrm{msec}$ faster than changed-level trials for local level repetitions and $32 \mathrm{msec}$ faster for global level repetitions $(F<1)$. There was no suggestion in the data that contrast balancing reduced the effect of level repetition. The frequency content $\times$ level repetition interaction was nonsignificant $(F<1)$, and responses were faster in the repeated-level than in the changed-level condition for contrast-balanced stimuli $[F(1,31)=22.26, p<.001]$, as well as for bright ones $[F(1,31)=24.24, p<.001]$. Thus, there was no indication that attention to local and global forms, as measured by the level-repetition effect, was impaired by the elimination of low spatial frequencies.

One purpose of the present experiment was to test the hypothesis that the level-repetition effect might occur because adjustments in the diameter of the attended area are carried over from one trial to the next. We argued that correct adjustment of the diameter of the attentional

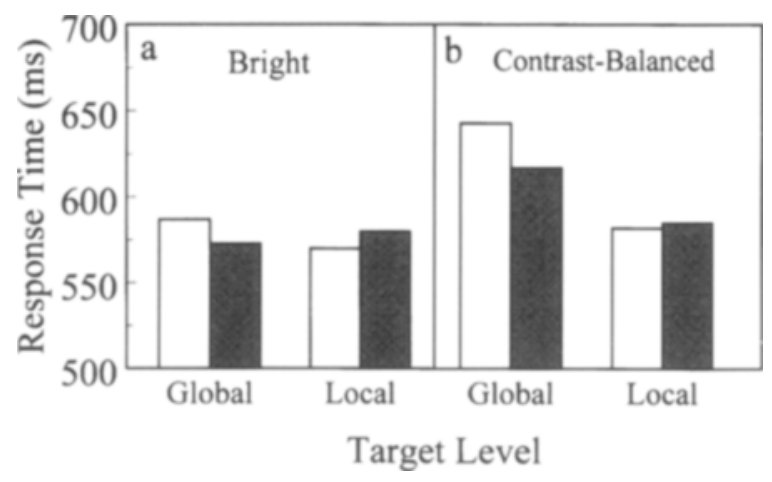

Figure 6. Mean response time (in milliseconds) to global and local targets for stimuli presented in the upper visual field (open bars) and lower visual field (filled bars). Data are presented separately for bright (a) and contrast-balanced (b) stimuli. 
Table 2

Mean Response Time (in Milliseconds) and Percentage Errors for Each Level Repetition, Target Repetition, and Field Repetition as a Function of Level and Frequency Content

\begin{tabular}{|c|c|c|c|c|c|c|c|c|c|c|}
\hline \multirow[b]{3}{*}{$\begin{array}{l}\text { Level } \\
\text { rors }\end{array}$} & \multirow[b]{3}{*}{ Target } & \multirow[b]{3}{*}{ Field } & \multicolumn{4}{|c|}{ Bright } & \multicolumn{4}{|c|}{ Contrast Balanced } \\
\hline & & & \multicolumn{2}{|c|}{ Global } & \multicolumn{2}{|c|}{ Local } & \multicolumn{2}{|c|}{ Global } & \multicolumn{2}{|c|}{ Local } \\
\hline & & & RT & Errors & RT & Errors & RT & Errors & RT & $\mathrm{E} r$ \\
\hline \multirow[t]{4}{*}{ Repeated } & repeated & repeated & 577 & 0.8 & 585 & 4.7 & 606 & 4.7 & 602 & 4.3 \\
\hline & & changed & 617 & 4.7 & 646 & 6.3 & 649 & 6.3 & 635 & 13.3 \\
\hline & changed & repeated & 594 & 2.7 & 592 & 4.3 & 681 & 6.6 & 601 & 6.6 \\
\hline & & changed & 595 & 2.7 & 588 & 3.9 & 642 & 5.1 & 606 & 4.3 \\
\hline \multirow[t]{4}{*}{ Changed } & repeated & repeated & 622 & 5.5 & 601 & 4.7 & 684 & 7.0 & 620 & 6.3 \\
\hline & & changed & 627 & 10.2 & 662 & 10.9 & 701 & 10.6 & 676 & 11.3 \\
\hline & changed & repeated & 647 & 4.3 & 639 & 8.6 & 673 & 3.5 & 643 & 9.4 \\
\hline & & changed & 618 & 1.6 & 639 & 3.5 & 648 & 3.1 & 627 & 8.6 \\
\hline
\end{tabular}

"spotlight" should be beneficial only if the "spotlight" is also directed at the correct location. This hypothesis thus predicts that the level-repetition effect will be large on trials in which the level repetition co-occurs with field repetition but will be eliminated, or at least drastically reduced, on trials in which level and field repetition do not co-occur. The present data provide little support for this hypothesis. The level-repetition effect was slightly larger on repeated-field $(36 \mathrm{msec})$ than on changed-field $(28 \mathrm{msec})$ trials, but the level repetition $\times$ field repetition interaction was nonsignificant $(F<1)$, and the level-repetition effect was significant on changed-field trials $[F(1,31)=9.44, p<.01]$, as well as on repeated-field trials $[F(1,31)=31.38, p<.001]$.

The main effect of target repetition was nonsignificant $(F<1)$, (see Figure $7 \mathrm{~b}$ ). In contrast, the main effect of field repetition was significant $[F(1,31)=10.56, p<$ $.01]$. Performance was better when the stimulus appeared in the same visual field on trials $n-1$ and $n$ than when it did not (see Figure 7c). However, there was also a significant field repetition $\times$ target repetition interaction $[F(1,31)=28.23, p<.001]$ (see Figure 8). This interaction occurred because field repetition improved performance when the target letter also was repeated $[F(1,31)=53.81, p<.001]$, but seems to have harmed

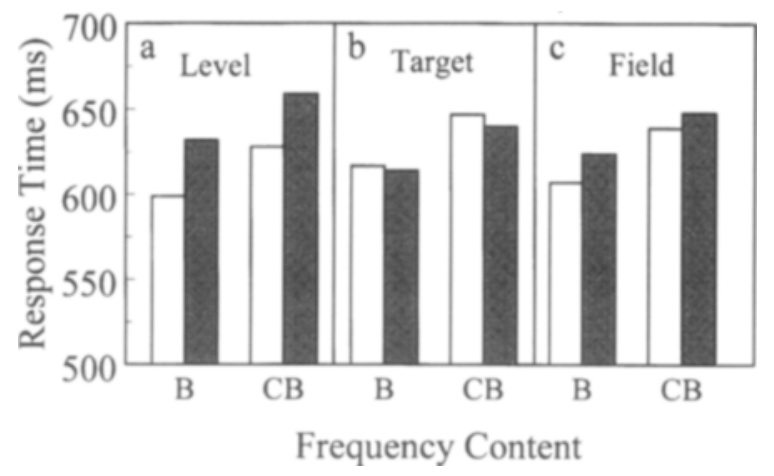

Figure 7. Mean response time (in milliseconds) to bright (B) and contrast-balanced (CB) stimuli on repeated-level (open bars) and changed-level (filled bars) trials (a), on repeated-target (open bars) and changed-target (filled bars) trials (b), and on repeatedfield (open bars) and changed-field (filled bars) trials (c). performance when the target letter changed $[F(1,31)=$ $3.54, p<.07]$. Examined from the other perspective, there was a small but significant improvement in performance with target repetition when the stimulus appeared in the same visual field on trials $n-1$ and $n[F(1,31)=$ $6.79, p<.05]$, but target repetition harmed performance when the stimulus location changed $[F(1,31)=23.92$, $p<.001$ ] (see Figure 8).

The level repetition $\times$ target repetition interaction was nonsignificant $(F<1)$, but the frequency content $\times$ level repetition $\times$ target repetition interaction was significant $[F(1,31)=9.41, p<.01]$. This interaction reflects the fact that for bright stimuli, the size of the level-repetition effect tended to be smaller when the target letter also repeated than when it did not $[F(1,31)=5.26, p<.05]$ (see Figure 9a), whereas the reverse was true for contrast-balanced stimuli $[F(1,31)=4.91, p<.05]$ (see Figure 9b). Nevertheless, the level-repetition effect was significant at all levels of target repetition for both bright and contrast-balanced stimuli. Level repetition facilitated performance for bright repeated targets $[F(1,31)=7.92, p<.01]$, bright changed targets $[F(1,31)=26.55, p<.001]$, contrast-balanced repeated targets $[F(1,31)=20.27, p<.001]$, and contrast-balanced changed targets $[F(1,31)=24.88, p<.001]$ (see Figure 9).

Finally, the target level $\times$ level repetition $\times$ target repetition interaction was significant $[F(1,31)=9.92, p<$ $.01]$. This interaction reflects the fact that for global targets, the size of the level-repetition effect tended to be larger when the target letter also repeated than when it did not, whereas the reverse was true for local targets.

\section{DISCUSSION}

Consistent with previous research (Badcock et al., 1990; LaGasse, 1993; Lamb \& Yund, 1993, in press), the present experiment shows that low spatial frequencies facilitate the processing of global forms. Eliminating low spatial frequencies by contrast balancing slowed RTs to global but not to local forms. This suggests that the frequently reported RT advantage for global forms reflects a temporal processing advantage provided by low spatial frequencies. .

Also consistent with previous data (Hughes et al., 1990; Lamb \& Yund, 1993, in press), the amount of interference 


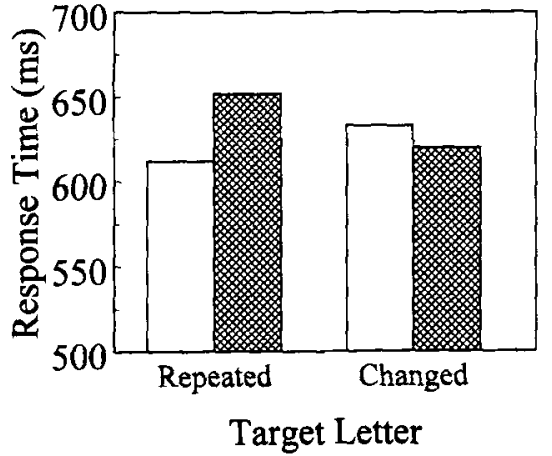

Figure 8. Mean response time (in milliseconds) on repeatedfield (open bars) and changed-field (filled bars) trials. Data are presented separately for repeated-target and changed-target trials.

for contrast-balanced stimuli was at least as great as that observed for bright stimuli. The fact that interference occurred even when low spatial frequencies had been removed from the stimulus lends no support to the hypothesis that interference between local and global forms stems from inhibitory interactions between high and low spatial frequency channels.

The present results were consistent with Previc's (1990) hypothesis that the upper visual field is specialized for local analysis, whereas the lower visual field is specialized for global analysis. The effects, though small, were in the same direction as those found by Christman (1993).

The field-repetition effect observed in the present experiment is consistent with the idea that adjustments of an attentional "spotlight" carry over from one trial to the next so that performance is facilitated if the stimulus appears in the same location on consecutive trials (see Figure 7). If so, however, the beneficial effect of attending to the correct location was overwhelmed by the detrimental effect of a change in the target letter, since the field-repetition effect was absent on changed-target trials (see Figure 8). On the other hand, the interaction between field and target repetition is also consistent with the "bypass rule" (Fletcher \& Rabbitt, 1978; Krueger \& Shapiro, 1981). Field repetition facilitated performance only on trials in which the same response was required on trials $n-1$ and $n$ (i.e., repeated-target trials).

There was also an indication that the "bypass rule" might be operating to some extent when the changing stimulus attribute was target level. The interaction between level and target repetition with contrast-balanced stimuli is in the direction predicted by the "bypass rule" (see Figure 9b). However, the pattern of RTs for bright stimuli did not conform to the "bypass rule," because in this case changing the level of the target did not bias subjects to change their response (see Figure 9a). In addition, level repetition significantly facilitated performance regardless of whether or not the target letter repeated, for both bright and contrast-balanced stimuli. Thus the level-repetition effect cannot be accounted for by the "bypass rule."
The present experiment provides no support for the idea that attention to local versus global forms is based on spatial frequency. Eliminating low spatial frequencies from the stimulus produced no measurable change in the level-repetition effect. Thus spatial frequency does not seem to be the basis for attentional selection between local and global forms, whether measured by the levelrepetition effect (the present experiment), or by the "biasing" procedure (Lamb \& Yund, 1993).

The present data also lend little support to the hypothesis that the level-repetition effect reflects changes in the diameter of an attentional "spotlight" which are carried over from one trial to the next. Having the "spotlight" adjusted to the appropriate size would seem to be of value only if the stimulus occurred in the attended area. However, the magnitude of the level-repetition effect did not depend on a co-occurrence of level and field repetition. Of course, it is possible that adjustments to the location of the "spotlight" could be fast and efficient relative to adjustments in the diameter of the "spotlight." If so, the beneficial effect of level repetition (having the diameter of the "spotlight" adjusted appropriately) might far outweigh the beneficial effect of field repetition (having the location of the "spotlight" appropriately adjusted) and thus account for the lack of an interaction between level and field repetition.

Even if one were to accept this assumption, however, other aspects of the data are not consistent with the hypothesis that level repetition reflects changes in the diameter of an attentional "spotlight." If one assumes that the analysis of everything within the attended region is facilitated (relative to a neutral baseline), then both local and global forms should be facilitated by a large-diameter spotlight, because forms at both levels will fall within the attended area. In contrast, only local forms should be facilitated by a small-diameter spotlight, because the global form would fall outside the attended area. Such asymmetric attentional effects were found in a study by Robertson et al. (1993). However, the level-repetition effect in the present experiment was not different for local and global forms. Thus it seems unlikely that the effect

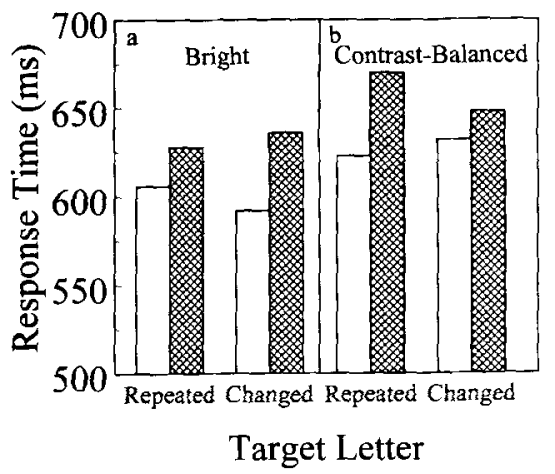

Figure 9. Mean response time (in milliseconds) to bright (a) and contrast-balanced (b) stimuli for repeated-level (open bars) and changed-level (filled bars) trials. Data are presented separately for repeated-target and changed-target trials. 
of level repetition in the present experiment resulted from variations in the diameter of an attentional "spotlight."

Another possibility is that the effect of level repetition is an example of object priming. An internal representation of target characteristics on trial $n-1$ might be formed which then facilitates identification of a target occurring at that same level on trial $n$. A potential problem with this hypothesis is that the level-repetition effect occurred in the absence of a target-repetition effect. In order to account for this fact, one would have to assume that information regarding target level was carried over from one trial to the next in the absence of any information regarding target identity. Although it might seem that any representation that would code target level would also include information about target identity, Biederman and Cooper (1992) have provided evidence that the mechanism responsible for representing an object's shape is separate from the mechanism that is responsible for representing its size. Thus, it is possible that target identity and target level are represented separately and that only the latter information carries over from one trial to the next to produce a repetition effect.

A hypothesis that escapes this difficulty altogether, however, is that a level-specific mechanism (rather than level-specific information) is "primed" by level repetition. There is neuropsychological and electrophysiological evidence that efficient global analysis and efficient local analysis are associated with different brain regions (Delis, Robertson; \& Efron, 1986; Heinz \& Munte, 1993; Lamb et al., 1989, 1990; Robertson et al., 1988). If efficient analysis of local versus global forms involves the operation of somewhat different sets of mechanisms, the level-repetition effect might occur because the appropriate mechanism is already activated on trial $n$ as the result of just having performed an analysis of a form at that same level on trial $n-1$. Such "mechanism" priming would not require that any representation of target characteristics be carried over from one trial to the next, so that the finding of a level-repetition effect in the absence of a target-repetition effect would be expected.

Either of these priming hypotheses could account for the "categorical" attention effects described by Robertson et al. (1993), but the present data are not consistent with the conjecture that "categorical" attention is based on spatial frequency, since the level-repetition effect was unaffected by contrast balancing. The hypothesis that "categorical" attention might be based on spatial frequency suffers on logical as well as empirical grounds. The idea behind "categorical" attention is that information at the attended level is selected out for preferential analysis on the basis of some characteristic that distinguishes local from global forms. However, while a range of low spatial frequencies could be selected which would be sufficient to identify global but not local forms, high spatial frequencies sufficient to identify local forms would always be sufficient to identify global ones as well. That is, attentional selection based on spatial frequency should be asymmetric, with global but not local information being made available when selection is based on low spatial frequencies, but with both local and global information being available from the selection of high spatial frequencies. This is not consistent with the present data in that the level-repetition effect did not differ as a function of level, nor is it consistent with numerous other studies finding symmetric "attentional biasing" effects between local and global forms (Kinchla et al., 1983; Lamb \& Robertson, 1987; Lamb \& Yund, 1993; Robertson et al., 1993; Robertson et al., 1988). The one clear case of asymmetric attentional effects was in the opposite direction to that predicted by the spatial frequency hypothesis (Robertson et al., 1993). In that study, local biasing facilitated local identification at the expense of global, while global biasing facilitated both global and local identification. This finding was thought to be due to changes in the diameter of an attentional "spotlight," so-called "regional" attention.

As stated earlier, the "regional" attention hypothesis has difficulty accounting for the present level-repetition effect. This hypothesis also has some difficulty accounting for the data of a number of studies showing levelspecific attention effects between stimuli at different spatial locations (Briand, 1993, 1994; Paquet \& Merikle, 1988). The priming hypotheses proposed here more easily accommodate these findings, as well as the present data, because their functioning does not depend on spatial location. The priming hypotheses can also account for the performance tradeoffs observed with the "biasing" procedure used by many investigators (Kinchla et al., 1983; Lamb \& Robertson, 1987; Lamb \& Yund, 1993; Robertson et al., 1993; Robertson et al., 1988), because level repetitions would occur frequently for the biased but not for the unbiased level.

In summary, there is evidence that at least two different mechanisms can modulate the analysis of hierarchical levels of structure. Some data are consistent with the hypothesis that variations in the diameter of an attentional "spotlight" modulate the analysis of local and global forms (Lamb \& Robertson, 1988; Robertson et al., 1993). The present data are not easily accommodated by this hypothesis, but are well accounted for by a hypothesis in which level repetition facilitates performance by activation of level-specific mechanisms. This hypothesis can also account for the results of the majority of studies examining attentional selection between local and global forms. The present data provide no support for the hypothesis that such attentional effects are based on spatial frequency.

\section{REFERENCES}

Badcock, J. C., W'hitworth, F. A., Badcock, D. R., \& Lovegrove, W. J. (1990). Low-frequency filtering and the processing of localglobal stimuli. Perception, 19, 617-629.

Bertelson, P. (1965). Serial choice reaction-time as a function of response versus signal-and-response repetition. Nature, 206, 21 7-218.

Biederman, I., \& Cooper, E. E. (1992). Size invariance in visual object priming. Journal of Experimental Psychology: Human Perception \& Performance, 18, 121-133.

BRIAND, K. A. (1993). Efficient filtering of irrelevant global and local 
information when target level and location are random. Psychological Research, 55, 264-269.

BRIAND, K. A. (1994). Selective attention to global and local structure of objects: Alternative measures of nontarget processing. Perception \& Psychophysics, $\mathbf{5 5}, 562-574$.

Christman, S. D. (1993). Local-global processing in the upper versus lower visual fields. Bulletin of the Psychonomic Society, 31, 275278.

Delis, D. C., Robertson, L. C., \& Efron, R. (1986). Hemispheric specialization of memory for visual hierarchical stimuli. Neuropsychologia, 24, 205-214.

De Valors, R. L., \& De Valois, K. K. (1990). Spatial vision. New York: Oxford University Press.

Filoteo, J. V., Delis, D. C., Demadura, T. L., Salmon, D. P., Roman, M. J., \& Shults, C. W. (1994). Abnormally rapid disengagement of covert attention to global and local stimulus levels may underlie the visuoperceptual impairment in Parkinson's patients. Neuropsychology, 8, 210-217.

Filoteo, J. V., Delis, D. C., Massman, P. J., Demadura, T. L., \& ButTERS, N. (1992). Directed and divided attention in Alzheimer's disease: Impairment in shifting of attention to global and local stimuli. Journal of Clinical \& Experimental Psychology, 14, 871-883.

FLETCHeR, B., \& RABBITT, P. M. A. (1978). The changing pattern of perceptual analytic strategies and response selection with practice in a two-choice reaction time task. Quarterly Journal of Experimental Psychology, 30, 417-427.

HeinZ, H. J., \& MunTE, T. F. (1993). Electrophysiological correlates of hierarchical stimulus processing: Dissociation between onset and later stages of global and local target processing. Neuropsychologia, 31, $841-852$.

Hughes, H. C. (1986). Asymmetric interference between components of suprathreshold compound gratings. Perception \& Psychophysics, 40, $241-250$.

Hughes, H. C., Fendrich, R., \& REuter-LoRenz, P. A. (1990). Global versus local processing in the absence of low spatial frequencies. Journal of Cognitive Neuroscience, 2, 272-282.

Hughes, H. C., Layton, W. M., Baird, J. C., \& Lester, L. S. (1984). Global precedence in visual pattern recognition. Perception \& Psychophysics, 35, 361-371.

Kinchla, R. A., Solis-Macias, V., \& Hoffman, J. (1983). Attending to different levels of structure in a visual image. Perception \& Psychophysics, 33, 1-10.

Kitterle, F. L., Christman, S., \& Conesa, J. (1993). Hemispheric differences in the interference among components of compound gratings. Perception \& Psychophysics, 54, 785-793

KRUEGER, L. E., \& ShaPIRo, R. G. (1981). Intertrial effects of samedifferent judgements. Quarterly Journal of Experimental Psychology, 33A, 24l-265.

LAGASSE, L. L. (1993). Effects of good form and spatial frequency on global precedence. Perception \& Psychophysics, 53, 89-105.

LAMB, M. R., \& RoBERTSON, L. C. (1987). Effects of acute alcohol on attention and the processing of hierarchical patterns. Alcoholism. Clinical \& Experimental Research, 11, 243-248.

LAMB, M. R., \& ROBERTSON, L. C. (1988). The processing of hierarchical stimuli: Effects of retinal locus, locational uncertainty, and stimulus identity. Perception \& Psychophysics, 44, 172-181.

LAMB, M. R., \& ROBERTSON, L. C. (1989). Do response time advantage and interference reflect the order of processing of global- and locallevel information? Perception \& Psychophysics, 46, 254-258.

LAMB, M. R., \& ROBERTSON, L. C. (1990). The effect of visual angle on global and local reaction times depends on the set of visual angles presented. Perception \& Psychophysics, 47, 489-496.

LAMB, M. R., ROBERTSON, L. C., \& KNIGHT, R. T. (1989). Attention and interference in the processing of global and local information: Effects of unilateral temporal-parietal junction lesions. Neuropsychologia, 27, 471-483.

Lamb, M. R., Robertson, L. C., \& KNIGHT, R. T. (1990). Component mechanisms underlying the processing of hierarchically organized patterns: Inferences from patients with unilateral cortical lesions. Journal of Experimental Psychology: Learning, Memory, \& Cognition, 16, 471-483.

LAMB, M. R., \& YUND, E. W. (1993). The role of spatial frequency in the processing of hierarchically organized structure. Perception \& Psychophysics, 54, 773-784.

LAMB, M. R., \& YUND, E. W. (in press). Spatial frequency and interference between global and local levels of structure. Visual Cognition.

Lovegrove, W., \& PePper, K. (1994). The influence of low-level processing in the global precedence effect. In S. Ballesteros (Ed.), Cog nitive approaches to human perception (pp. 71-90). Hillsdale, $\mathrm{NJ}$ : Erlbaum.

Miller, J. (1981). Global precedence in attention and decision. Journal of Experimental Psychology: Human Perception \& Perfor mance, 7, 1161-1174.

NAvon, D. (1977). Forest before trees: The precedence of global features in visual perception. Cognitive Psychology, 9, 353-383.

Navon, D. (1991). Testing a queue hypothesis for the processing of global and local information. Journal of Experimental Psychology General, 120, 173-189.

Navon, D., \& Norman, J. (1983). Does global precedence really depend on visual angle? Journal of Experimental Psychology: Human Perception \& Performance, 9, 955-965.

PAQUET, L. (1992). Global and local processing in nonattended objects: A failure to induce local processing dominance. Journal of Experimental Psychology: Human Perception \& Performance, 18, 512529

Paquet, L., \& Merikle, P. M. (1988), Global precedence in attended and unattended objects. Journal of Experimental Psychology: Human Perception \& Performance, 14, 89-100.

Pomerantz, J. R. (1983). Global and local precedence: Selective attention in form and motion perception. Journal of Experimental Psychology: General, 112, 516-540.

PREVIC, F. (1990). Functional specialization in the lower and upper visual fields in humans: Its ecological origins and neurophysiological implications. Behavioral \& Brain Sciences, 13, 519-575.

Robertson, L. C., Egly, R., Lamb, M. R., \& Kerth, L. (1993). Spatial attention and cuing to global and local levels of hierarchical structure. Journal of Experimental Psychology: Human Perception \& Performance, 19, 471-487.

Robertson, L. C., \& LAMB, M. R. (1991). Neuropsychological perspectives on theories of part/whole organization. Cognitive Psychology, 23, 299-330.

RobertSON, L. C., LAMB, M. R., \& KNIGHT, R. T. (1988). Effects of lesions of temporal-parietal junction on perceptual and attentional processing in humans. Journal of Neuroscience, 8, 3757-3769.

SERGENT, J. (1982). The cerebral balance of power: Confrontation or cooperation? Journal of Experimental Psychology: Human Perception \& Performance, 8, 252-272.

SERGENT, J. (1987). Failures to confirm the spatial-frequency hypothesis: Fatal blow or healthy complication? Canadian Journal of Psychology, 41, 412-428.

Shulman, G. L., Sullivan, M. A., Gish, K., \& Sakoda, W. J. (1986) The role of spatial-frequency channels in the perception of local and global structure. Perception, 15, 259-273.

Shulman, G. L., \& Wilson, J. (1987). Spatial frequency and selective attention to local and global information. Perception, 16, 89-101.

TERrY, K. M., VALDES, L. A., \& NeILL, W. T. (1994). Does "inhibition of return" occur in discrimination tasks? Perception \& Psychophysics, 55, 279-286.

WARD, L. M. (1982). Determinants of attention to local and global features of visual forms. Journal of Experimental Psychology: Human Perception \& Performance, 8, 562-585.

(Manuscript received July 27, 1994; revision accepted for publication August 2, 1995.) 